TRANSACTIONS OF THE

AMERICAN MATHEMATICAL SOCIETY

Volume 358, Number 5 , Pages 2269-2289

S 0002-9947(05)03853-5

Article electronically published on December 20, 2005

\title{
RESONANCE, LINEAR SYZYGIES, CHEN GROUPS, AND THE BERNSTEIN-GELFAND-GELFAND CORRESPONDENCE
}

\author{
HENRY K. SCHENCK AND ALEXANDER I. SUCIU
}

\begin{abstract}
If $\mathcal{A}$ is a complex hyperplane arrangement, with complement $X$, we show that the Chen ranks of $G=\pi_{1}(X)$ are equal to the graded Betti numbers of the linear strand in a minimal, free resolution of the cohomology ring $A=H^{*}(X, \mathbb{k})$, viewed as a module over the exterior algebra $E$ on $\mathcal{A}$ :
\end{abstract}

$$
\theta_{k}(G)=\operatorname{dim}_{\mathbb{k}} \operatorname{Tor}_{k-1}^{E}(A, \mathbb{k})_{k}, \quad \text { for } k \geq 2,
$$

where $\mathbb{k}$ is a field of characteristic 0 . The Chen ranks conjecture asserts that, for $k$ sufficiently large, $\theta_{k}(G)=(k-1) \sum_{r \geq 1} h_{r}\left(\begin{array}{c}r+k-1 \\ k\end{array}\right)$, where $h_{r}$ is the number of $r$-dimensional components of the projective resonance variety $\mathcal{R}^{1}(\mathcal{A})$. Our earlier work on the resolution of $A$ over $E$ and the above equality yield a proof of the conjecture for graphic arrangements. Using results on the geometry of $\mathcal{R}^{1}(\mathcal{A})$ and a localization argument, we establish the inequality

$$
\theta_{k}(G) \geq(k-1) \sum_{r \geq 1} h_{r}\left(\begin{array}{c}
r+k-1 \\
k
\end{array}\right), \quad \text { for } k \gg 0,
$$

for arbitrary $\mathcal{A}$. Finally, we show that there is a polynomial $\mathrm{P}(t)$ of degree equal to the dimension of $\mathcal{R}^{1}(\mathcal{A})$, such that $\theta_{k}(G)=\mathrm{P}(k)$, for all $k \gg 0$.

\section{INTRODUCTION}

1.1. Orlik-Solomon algebra. Let $\mathcal{A}=\left\{H_{1}, \ldots, H_{n}\right\}$ be an arrangement of complex hyperplanes in $\mathbb{C}^{\ell}$. A fundamental question in the subject is to decide whether a given topological invariant of the complement, $X(\mathcal{A})=\mathbb{C}^{\ell} \backslash \bigcup_{H \in \mathcal{A}} H$, is determined by the intersection lattice, $L(\mathcal{A})=\left\{\bigcap_{H \in \mathcal{A}^{\prime}} H \mid \mathcal{A}^{\prime} \subseteq \mathcal{A}\right\}$, and, if so, to find an explicit combinatorial formula for such an invariant.

For example, in 26, Orlik and Solomon showed that the cohomology ring of the complement is entirely determined by $L(\mathcal{A})$. More precisely, the Orlik-Solomon algebra $A=H^{*}(X(\mathcal{A}), \mathbb{Z})$ is the quotient of the exterior algebra $E=\bigwedge\left(\mathbb{Z}^{n}\right)$ on generators $e_{1}, \ldots, e_{n}$ in degree 1 by the ideal $I$ generated by all elements of the form $\partial e_{i_{1} \ldots i_{r}}:=\sum_{q}(-1)^{q-1} e_{i_{1}} \cdots \widehat{e_{i_{q}}} \cdots e_{i_{r}}$, for which codim $H_{i_{1}} \cap \cdots \cap H_{i_{r}}<r$. Notice that $I$ is generated in degrees 2 and higher; in particular, $A_{0}=E_{0}=\mathbb{Z}$ and $A_{1}=E_{1}=\mathbb{Z}^{n}$.

For each element $a \in A_{1}$, the Orlik-Solomon algebra can be turned into a cochain complex $(A, a)$. The $i$ th term of this complex is simply the degree $i$ graded piece

Received by the editors January 31, 2004 and, in revised form, August 17, 2004.

2000 Mathematics Subject Classification. Primary 16E05, 52C35; Secondary 13D07, 20F14.

Both authors were supported by NSF Collaborative Research grant DMS 03-11142; the first author was also supported by NSA grant MDA 904-03-1-0006 and ATP grant 010366-0103. 
of $A$, and the differential is given by multiplication by $a$ :

$$
(A, a): \quad 0 \longrightarrow A_{0} \stackrel{a}{\longrightarrow} A_{1} \stackrel{a}{\longrightarrow} A_{2} \stackrel{a}{\longrightarrow} \cdots \stackrel{a}{\longrightarrow} A_{\ell} \longrightarrow 0 .
$$

This complex arose in the work of Aomoto [1] on hypergeometric functions, and in the work of Esnault, Schechtman and Viehweg [15] on cohomology with coefficients in local systems. In [33, Yuzvinsky showed that, for generic $a$, the complex (1.1) is exact.

1.2. Resonance varieties. In [16, Falk initiated the study of the cohomology jumping loci for an arrangement complement. Fix a field $\mathbb{k}$; abusing notation, we will also denote by $A=H^{*}(X(\mathcal{A}), \mathbb{k})$ the Orlik-Solomon algebra over $\mathbb{k}$. The resonance varieties of $\mathcal{A}$ are the loci of points $a=\sum_{i=1}^{n} a_{i} e_{i} \leftrightarrow\left(a_{1}: \cdots: a_{n}\right)$ in $\mathbb{P}\left(A_{1}\right) \cong \mathbb{P}^{n-1}$ for which $(A, a)$ fails to be exact. More precisely, for each $k \geq 1$,

$$
\mathcal{R}^{k}(\mathcal{A})=\left\{a \in \mathbb{P}^{n-1} \mid H^{k}(A, a) \neq 0\right\} .
$$

The resonance varieties of $\mathcal{A}$ lie in the hyperplane $\sum_{i=1}^{n} a_{i}=0$, and depend only on the lattice-isomorphism type of $L(\mathcal{A})$. In [16, Falk also introduced the notion of a neighborly partition: a partition $\Pi$ of $\mathcal{A}$ is neighborly if, for any rank two flat $Y \in L_{2}(\mathcal{A})$ and any block $\pi$ of $\Pi$,

$$
\mu(Y) \leq|Y \cap \pi| \Longrightarrow Y \subseteq \pi
$$

where $\mu$ denotes the Möbius function of the lattice. Now assume char $\mathbb{k}=0$. Falk showed that all components of $\mathcal{R}^{1}(\mathcal{A})$ arise from neighborly partitions of subarrangements of $\mathcal{A}$. In particular, each flat $Y \in L_{2}(\mathcal{A})$ gives rise to a "local" component of dimension $\mu(Y)-1$; see Example 2.3 for how this works.

Falk also conjectured that the components of $\mathcal{R}^{1}(\mathcal{A})$ are projective linear subspaces; this was proved in [8], and generalized to $\mathcal{R}^{k}(\mathcal{A})$ in [5]. Libgober and Yuzvinsky [22] showed that the components of $\mathcal{R}^{1}(\mathcal{A})$ are in fact disjoint, and positive-dimensional. These facts will be used in an essential way later in the paper. We note that the characteristic zero assumption is necessary; the aforementioned results depend on this. A thorough treatment of resonance varieties over arbitrary fields (and even commutative rings) can be found in a recent paper of Falk [17] (see also 24]).

1.3. Lower central series and Chen ranks. The fundamental group of the complement, $G(\mathcal{A})=\pi_{1}(X(\mathcal{A}))$, is not necessarily determined by the intersection lattice. Even so, the ranks of the lower central series quotients,

$$
\phi_{k}(G):=\operatorname{rank} G_{k} / G_{k+1},
$$

are combinatorially determined (here $G_{1}=G, G_{2}=G^{\prime}=[G, G]$, and $G_{k+1}=$ $\left.\left[G, G_{k}\right]\right)$. In fact, due to the formality of $X(\mathcal{A})$, the LCS ranks depend only on the $\mathbb{k}$-algebra $A=H^{*}(X(\mathcal{A}), \mathbb{k})$, where char $\mathbb{k}=0$. Explicit formulas for the LCS ranks of an arrangement group are available in some cases (most notably, when $L(\mathcal{A})$ is supersolvable), but a general, all-encompassing formula remains elusive. See [18, [31, 34] for surveys of the problem, and [30, 29] for recent developments.

More manageable topological invariants are the Chen ranks,

$$
\theta_{k}(G):=\phi_{k}\left(G / G^{\prime \prime}\right) \text {. }
$$

Introduced by K.T. Chen in his thesis [4, these are the LCS ranks of the maximal metabelian quotient of $G$. For example, if $F_{n}$ is the free group of rank $n$, then 
$\theta_{1}\left(F_{n}\right)=n$, and $\theta_{k}\left(F_{n}\right)=(k-1)\left(\begin{array}{c}k+n-2 \\ k\end{array}\right)$, for $k \geq 2$. The original motivation for the study of the invariants $\theta_{k}(G)$ came from the study of link groups; see Massey's paper [23] and the references therein.

The study of Chen ranks of arrangement groups was started in [6], 7]. The ranks $\theta_{k}(G(\mathcal{A}))$ were determined in a number of cases, including the pure braid groups. They proved to be quite subtle and useful invariants, distinguishing in some instances between arrangement groups with the same LCS ranks.

1.4. Resonance and Chen ranks. For about a decade, it was an open question whether the Chen ranks of an arrangement are combinatorially determined; see e.g. [18, §2.3]. This question was recently settled in the affirmative in [28. There remained the question of computing explicitly the Chen ranks of an arrangement group $G(\mathcal{A})$ in terms of the intersection lattice $L(\mathcal{A})$. Based on the work in [6], [7], a precise combinatorial formula for the Chen ranks was conjectured in 31]:

Conjecture A (Resonance formula for Chen ranks). Let $G=G(\mathcal{A})$ be an arrangement group, and let $h_{r}$ be the number of components of $\mathcal{R}^{1}(\mathcal{A})$ of dimension $r$. Then, for $k \gg 0$ :

$$
\theta_{k}(G)=(k-1) \sum_{r \geq 1} h_{r}\left(\begin{array}{c}
r+k-1 \\
k
\end{array}\right)
$$

In other words, $\theta_{k}(G)=\sum_{r \geq 1} h_{r} \theta_{k}\left(F_{r+1}\right)$. This formula can easily be verified for a pencil of $n$ lines (with $G=F_{n-1} \times \mathbb{Z}$ ), a near-pencil (with $G=F_{n-2} \times \mathbb{Z}^{2}$ ), or a product of such arrangements. Much less obviously, the conjecture holds for the braid arrangements [6], and for "decomposable" arrangements [7, 29]. Our methods give a unified proof of Conjecture $\mathrm{A}$ for all these classes of arrangements, and in fact apply more generally.

It was originally conjectured in 31 that equality (1.3) holds for all $k \geq 4$, but Example 6.3 below shows this is false. It turns out that the value for which $\theta_{k}(G)$ is given by a fixed polynomial in $k$ depends on the Castelnuovo-Mumford regularity of the linearized Alexander invariant.

1.5. Resonance and linear syzygies. In [30] we observed that there was a close connection between the resonance variety $\mathcal{R}^{1}(\mathcal{A})$ and the linear syzygies of $A$, where the Orlik-Solomon algebra $A$ is viewed as a module over the exterior algebra $E$. We define the linear strand in a minimal free resolution of $A$ over $E$ as the subcomplex of the form

$$
0 \longleftarrow A \longleftarrow E \longleftarrow E^{\beta_{12}}(-2) \longleftarrow E^{\beta_{23}}(-3) \longleftarrow \cdots \longleftarrow E^{\beta_{i, i+1}}(-i-1) \longleftarrow \cdots
$$

where $\beta_{i j}=\operatorname{dim}_{\mathbb{k}} \operatorname{Tor}_{i}^{E}(A, \mathbb{k})_{j}$. While $\beta_{12}$ is determined by the Möbius function of $L(\mathcal{A})$, a purely combinatorial formula for $\beta_{i, i+1}$ is unknown. However, many examples suggested to us that:

Conjecture B (Resonance formula for the linear strand). For $k \gg 0$, the graded Betti numbers of the linear strand are given by

$$
\beta_{k-1, k}=(k-1) \sum_{r \geq 1} h_{r}\left(\begin{array}{c}
r+k-1 \\
k
\end{array}\right) .
$$


1.6. Outline and results. We now outline the structure of paper, and state our main results.

We start in $₫ 2$ with a review of the linearized Alexander invariant $\mathfrak{B}$. First considered in [7, this graded module over the symmetric algebra $S=\operatorname{Sym}\left(A_{1}^{*}\right)$ is closely related to both the resonance variety $\mathcal{R}^{1}(\mathcal{A})$ [8, and to the Chen ranks $\theta_{k}(G)[28$.

In $\$ 3$, we express the linearized Alexander invariant as an Ext module. Key to our approach is the Bernstein-Gelfand-Gelfand correspondence, which gives (for our purposes) a relationship between linear complexes over the exterior algebra $E$ (on generators $e_{1}, \ldots, e_{n}$ ) and graded modules over the symmetric algebra $S$ (on generators $\left.x_{1}, \ldots, x_{n}\right)$. In Theorem 3.2 , we show that

$$
\mathfrak{B} \cong \operatorname{Ext}_{S}^{\ell-1}(F(A), S) .
$$

Here $F(A)$ is the top cohomology module of the Aomoto complex $(A, a)$ tensored with $S$, with $a=\sum_{i=1}^{n} x_{i} e_{i}$; cf. (3.3). The module $F(A)$ was first studied by Eisenbud, Popescu and Yuzvinsky in 14. The formulation of the BGG correspondence in 13 , shows that the local cohomology modules of $F(A)$ determine the free resolution of $A$ over $E$.

As a first application, we prove in Corollary 3.3 that Conjecture $\mathrm{A}$ and Conjecture B are equivalent:

$$
\theta_{k}(G)=\beta_{k-1, k}, \quad \text { for all } k \geq 2 .
$$

As another application, we prove in Theorem 3.4 that the Chen ranks conjecture holds for graphic arrangements. If $\Gamma$ is a graph and $\mathcal{A}=\mathcal{A}(\Gamma)$ the corresponding arrangement, we show that

$$
\theta_{k}(G)=(k-1)\left(\kappa_{2}+\kappa_{3}\right), \quad \text { for all } k \geq 3,
$$

where $\kappa_{s}$ is the number of cliques of size $s+1$. On the other hand, the components of $\mathcal{R}^{1}(\mathcal{A})$ are all projective lines, and there is one component for each triangle or complete quadrangle in $\Gamma$. Thus, formula (1.7) agrees with (1.3).

In $\$ 4$ we use the results of [8, 22, 28] and some commutative algebra to prove that the Chen ranks have polynomial growth, controlled by the resonance variety: there exists a polynomial $\mathrm{P}(t) \in \mathbb{Q}[t]$, of degree equal to the dimension of $\mathcal{R}^{1}(\mathcal{A})$, such that $\theta_{k}(G)=\mathrm{P}(k)$, for all $k \gg 0$. In particular, this implies

$$
\lim _{k \rightarrow \infty} \frac{\theta_{k}(G)}{(k-1) \sum_{r \geq 1} h_{r}\left(\begin{array}{c}
r+k-1 \\
k
\end{array}\right)} \in \mathbb{Q} .
$$

As an easy corollary of this, we compute the complexity of the Orlik-Solomon algebra $A$, viewed as a module over $E$, in the case when $\ell=3$.

In \$5, we use a localization argument to give a (sharp) lower bound on the Chen ranks, thereby proving one direction of Conjecture $\mathrm{A}$. For an arbitrary arrangement group $G$, we show (in Corollary 5.6) that

$$
\theta_{k}(G) \geq(k-1) \sum_{r \geq 1} h_{r}\left(\begin{array}{c}
r+k-1 \\
k
\end{array}\right), \quad \text { for all } k \gg 0 .
$$

In 86 , we give some examples illustrating the fact that the Chen ranks formula does not hold for small values of $k$. This phenomenon can be interpreted in terms of certain local cohomology modules, which reflect subtle combinatorial behavior in $L(\mathcal{A})$. 


\section{DRAMATIS PERSONAE}

Let $\mathcal{A}$ be an arrangement of complex hyperplanes in $\mathbb{C}^{\ell}$, with complement $X(\mathcal{A})$. Since we are primarily interested in the fundamental group $G(\mathcal{A})=\pi_{1}(X(\mathcal{A})$ ), we may restrict our attention to affine line arrangements in $\mathbb{C}^{2}$. Indeed, if $\mathcal{A}$ is an arbitrary arrangement, let $\mathcal{A}^{\prime}$ be a generic two-dimensional section of $\mathcal{A}$. Then, by the Lefschetz-type theorem of Hamm and Lê [21, the inclusion $X\left(\mathcal{A}^{\prime}\right) \hookrightarrow X(\mathcal{A})$ induces an isomorphism $\pi_{1}\left(X\left(\mathcal{A}^{\prime}\right)\right) \stackrel{\simeq}{\rightarrow} \pi_{1}(X(\mathcal{A}))$.

Now, if $\mathcal{A}$ is an (affine) line arrangement in $\mathbb{C}^{2}$, the cone on $\mathcal{A}$ is a central plane arrangement in $\mathbb{C}^{3}$, with complement $X(\mathcal{A}) \times(\mathbb{C} \backslash 0)$, and fundamental group $G(\mathcal{A}) \times \mathbb{Z}$; see 27 . In view of the above, we will assume throughout that $\mathcal{A}$ is a central arrangement. Most often, this will be an arrangement in $\mathbb{C}^{3}$, with projectivization a line arrangement in $\mathbb{P}^{2}$.

2.1. Chen groups and Alexander invariant. Let $G^{\prime}=[G, G]$ be the derived subgroup of $G$, and $G^{\prime \prime}=\left(G^{\prime}\right)^{\prime}$ the second derived subgroup. The group $G / G^{\prime}$ is the maximal abelian quotient of $G$, whereas $G / G^{\prime \prime}$ is its maximal metabelian quotient. The $k$ th Chen group of $G$ is, by definition, the $k$ th lower central series quotient of $G / G^{\prime \prime}$. Let $\theta_{k}(G)=\operatorname{rankgr}_{k}\left(G / G^{\prime \prime}\right)$ be its rank. For example, if $G=F_{n}$, the free group of rank $n$, then, as shown by Chen [4],

$$
\theta_{k}\left(F_{n}\right)=(k-1) \cdot\left(\begin{array}{c}
k+n-2 \\
k
\end{array}\right), \quad \text { for } k \geq 2 .
$$

Fix a basis $\left\{t_{1}, \ldots, t_{n}\right\}$ for $H_{1}(X)=G / G^{\prime}=\mathbb{Z}^{n}$, corresponding to meridians around the hyperplanes in $\mathcal{A}=\left\{H_{1}, \ldots, H_{n}\right\}$. This identifies the group ring $\mathbb{Z}\left[G / G^{\prime}\right]$ with the ring of Laurent polynomials $\Lambda=\mathbb{Z}\left[t_{1}^{ \pm 1}, \ldots, t_{n}^{ \pm 1}\right]$. Let $\mathfrak{I}$ be the augmentation ideal of $\Lambda$, and let gr $\Lambda$ be the graded ring associated to the $\mathfrak{I}$ adic filtration. The map $t_{i} \mapsto 1-x_{i}$ identifies this ring with the polynomial ring $S=\mathbb{Z}\left[x_{1}, \ldots, x_{n}\right]$.

The Alexander invariant, $B=B(\mathcal{A})$, is the abelian group $G^{\prime} / G^{\prime \prime}$, endowed with the $\Lambda$-module structure induced by the conjugation action in the extension $0 \rightarrow G^{\prime} / G^{\prime \prime} \rightarrow G / G^{\prime \prime} \rightarrow G / G^{\prime} \rightarrow 0$. As shown in [7, the module $B$ admits a finite presentation of the form

$$
\left(A_{2} \oplus E_{3}\right) \otimes \Lambda \stackrel{\Delta}{\longrightarrow} E_{2} \otimes \Lambda \longrightarrow B \longrightarrow 0 .
$$

Let $\operatorname{gr} B=\bigoplus_{k \geq 0} \mathfrak{I}^{k} B / \mathfrak{I}^{k+1} B$ be the associated graded module. A basic observation of W.S. Massey [23] asserts that $\operatorname{gr}_{k}\left(G / G^{\prime \prime}\right)=\operatorname{gr}_{k-2} B$, for $k \geq 2$. Consequently,

$$
\sum_{k \geq 0} \theta_{k+2} t^{k}=\operatorname{Hilb}(\text { gr } B \otimes \mathbb{k}),
$$

for any field $\mathbb{k}$ of characteristic 0 .

2.2. Linearized Alexander invariant. In general, the module $B$ is hard to compute, depending as it does on finding a (braid monodromy) presentation for the group $G$, and carrying out a laborious Fox calculus algorithm. The resulting presentation matrix $\Delta$ typically involves very complicated Laurent polynomials. On a conceptual level, it is not at all clear whether $B$ is combinatorially determined, since $G$ is not always determined by $L(\mathcal{A})$. For all these reasons, it is convenient to 
look at a simplified version of the Alexander invariant, which carries all the essential information we want to extract from this module.

The linearized Alexander invariant, $\mathfrak{B}=\mathfrak{B}(\mathcal{A})$, is the graded $S$-module presented by the "linearization" of the matrix $\Delta$ :

$$
\left(A_{2} \oplus E_{3}\right) \otimes S \stackrel{\Delta^{\operatorname{lin}}}{\longrightarrow} E_{2} \otimes S \longrightarrow \mathfrak{B} \longrightarrow 0 .
$$

The matrix $\Delta^{\text {lin }}$ appears in the statement of Theorem 4.6 and in Remark 4.7 from [8]; see also 24] and 28] for more general contexts. The reason for the terminology is as follows: Viewing $\Lambda \otimes \mathbb{C}$ as the coordinate ring of the algebraic torus $\left(\mathbb{C}^{*}\right)^{n}$ and $S \otimes \mathbb{C}$ as the coordinate ring of $\mathbb{C}^{n}$, the entries of $\Delta^{\text {lin }}$ are the derivatives at $1 \in\left(\mathbb{C}^{*}\right)^{n}$ of the corresponding entries of $\Delta$.

Let us describe the linearized Alexander matrix in a concrete fashion, following 8]. If $\delta_{i}$ denotes the $i$ th differential in the Koszul complex, then

$$
\Delta^{\mathrm{lin}}=\alpha_{2} \otimes \mathrm{id}+\delta_{3},
$$

where $\alpha_{2}$ is the adjoint of the canonical projection $\gamma_{2}: E_{2} \rightarrow A_{2}$, given by $\alpha_{2}(Y, j)=$ $e_{j} \wedge \sum_{i \in Y} e_{i}$, where $\left\{(Y, j) \mid Y \in L_{2}(\mathcal{A})\right.$ and $\left.j \in Y \backslash \min Y\right\}$ is the usual nbc-basis for $A_{2}$; see e.g. [27].

2.3. The module $\mathfrak{B}$, resonance, and Chen ranks. When $\mathbb{k}$ is a field of characteristic zero (which will be our standing assumption from now on), the linearized Alexander invariant $\mathfrak{B}:=\mathfrak{B} \otimes \mathbb{k}$ is related in a very concrete way to both the resonance variety $\mathcal{R}^{1}(\mathcal{A})$, and to the fundamental group $G=G(\mathcal{A})$.

The first important fact about the module $\mathfrak{B}$ is the identification of the variety defined by its annihilator ideal.

Theorem $2.1([\underline{8})$. The annihilator of the linearized Alexander invariant defines the resonance variety

$$
V(\operatorname{ann} \mathfrak{B})=\mathcal{R}^{1}(\mathcal{A}) .
$$

The second important fact about the module $\mathfrak{B}$ is the following linearized version of Massey's result.

Theorem $2.2([28])$. The Chen ranks, $\theta_{k}=\theta_{k}(G), k \geq 2$, are equal to the dimensions of the graded pieces of the linearized Alexander invariant

$$
\sum_{k \geq 2} \theta_{k} t^{k}=\operatorname{Hilb}(\mathfrak{B}) .
$$

In particular, the Chen ranks are combinatorially determined, and the Hilbert polynomial $\mathrm{P}(\mathfrak{B}, t) \in \mathbb{Q}[t]$ gives the asymptotic Chen ranks: for $k \gg 0, \theta_{k}=\mathrm{P}(\mathfrak{B}, k)$.

Example 2.3 (Braid arrangement). Let $\mathcal{A}$ be the braid arrangement in $\mathbb{P}^{2}$, with defining polynomial $Q=x y z(x-y)(x-z)(y-z)$. From the matroid (see Figure1), it is easy to see that the Orlik-Solomon algebra $A$ is the quotient of the exterior algebra $E$ on generators $e_{0}, \ldots, e_{5}$ by the ideal $I=\left\langle\partial e_{145}, \partial e_{235}, \partial e_{034}, \partial e_{012}, \partial e_{i j k l}\right\rangle$, where $i j k l$ runs over all four-tuples; it turns out that the elements $\partial e_{i j k l}$ are redundant.

The (minimal) free resolution of $A$ as a module over $E$ begins:

$$
0 \longleftarrow A \longleftarrow E \stackrel{\partial_{1}}{\longleftarrow} E^{4}(-2) \stackrel{\partial_{2}}{\longleftarrow} E^{10}(-3) \stackrel{\partial_{3}}{\longleftarrow} E^{15}(-4) \oplus E^{6}(-5) \longleftarrow \cdots,
$$



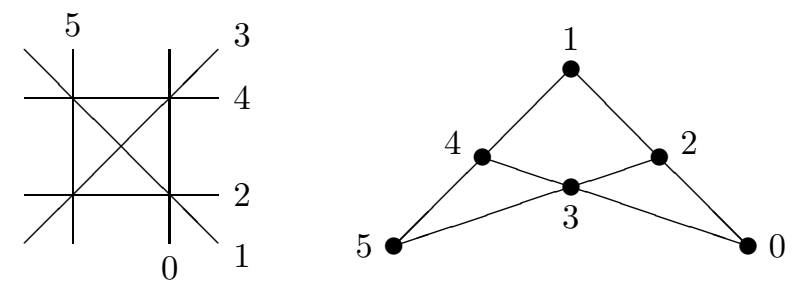

FIGURE 1. The braid arrangement and its matroid

where $\partial_{1}=\left(\begin{array}{llll}\partial e_{145} & \partial e_{235} & \partial e_{034} & \partial e_{012}\end{array}\right)$, and $\partial_{2}$ is equal to

$$
\left(\begin{array}{cccccccccc}
e_{1}-e_{4} & e_{1}-e_{5} & 0 & 0 & 0 & 0 & 0 & 0 & e_{3}-e_{0} & e_{2}-e_{0} \\
0 & 0 & e_{2}-e_{3} & e_{2}-e_{5} & 0 & 0 & 0 & 0 & e_{0}-e_{1} & e_{0}-e_{4} \\
0 & 0 & 0 & 0 & e_{0}-e_{3} & e_{0}-e_{4} & 0 & 0 & e_{1}-e_{5} & e_{2}-e_{5} \\
0 & 0 & 0 & 0 & 0 & 0 & e_{0}-e_{1} & e_{0}-e_{2} & e_{3}-e_{5} & e_{4}-e_{5}
\end{array}\right) .
$$

The resonance variety $\mathcal{R}^{1}(\mathcal{A}) \subset \mathbb{P}^{5}$ has 4 local components, corresponding to the triple points, and 1 essential component (i.e., one that does not come from any proper sub-arrangement), corresponding to the neighborly partition $\Pi=(05|13| 24)$ :

$$
\begin{aligned}
& \left\{x_{1}+x_{4}+x_{5}=x_{0}=x_{2}=x_{3}=0\right\},\left\{x_{2}+x_{3}+x_{5}=x_{0}=x_{1}=x_{4}=0\right\} \\
& \left\{x_{0}+x_{3}+x_{4}=x_{1}=x_{2}=x_{4}=0\right\},\left\{x_{0}+x_{1}+x_{2}=x_{3}=x_{4}=x_{5}=0\right\} \\
& \left\{x_{0}+x_{1}+x_{2}=x_{0}-x_{5}=x_{1}-x_{3}=x_{2}-x_{4}=0\right\} .
\end{aligned}
$$

The linearized Alexander invariant $\mathfrak{B}=\operatorname{coker}\left(\Delta^{\operatorname{lin}}: S^{31} \rightarrow S^{15}\right)$ is a module over the ring $S=\mathbb{k}\left[x_{0}, \ldots, x_{5}\right]$; the presentation matrix $\Delta^{\text {lin }}$ can be reduced by row and column operations to the matrix $\vartheta: S^{14} \rightarrow S^{4}$, with transpose

$$
\left(\begin{array}{cccc}
\sum x_{i} & 0 & 0 & 0 \\
0 & \sum x_{i} & 0 & 0 \\
0 & 0 & \sum x_{i} & 0 \\
0 & 0 & 0 & \sum x_{i} \\
x_{1}+x_{4}+x_{5} & 0 & 0 & 0 \\
0 & x_{2}+x_{3}+x_{5} & 0 & 0 \\
0 & 0 & x_{0}+x_{3}+x_{4} & 0 \\
0 & 0 & 0 & x_{0}+x_{1}+x_{2} \\
-x_{3} & 0 & 0 & x_{3} \\
0 & x_{4} & 0 & x_{4} \\
0 & 0 & -x_{5} & x_{5} \\
x_{0} & x_{0} & 0 & 0 \\
x_{2} & 0 & -x_{2} & 0 \\
0 & x_{1} & x_{1} & 0
\end{array}\right) .
$$

A computation shows that

$$
\operatorname{Hilb}(\mathfrak{B})=\left(4 t^{2}+2 t^{3}-t^{4}\right) /(1-t)^{2}=4 t^{2}+10 t^{3}+15 t^{4}+20 t^{5}+\cdots .
$$

Thus, $\theta_{1}=6, \theta_{2}=4$, and $\theta_{k}=5(k-1)$, for $k \geq 3$. This agrees with the computations in [6], and with the values predicted by Conjecture $\mathrm{A}$. A generalization will be given in Theorem 3.4 . 


\section{The Bernstein-Gelfand-Gelfand Correspondence And $H^{*}(A, a)$}

3.1. The BGG correspondence. Let $V$ be a finite-dimensional vector space over a field $\mathbb{k}$. In this section, we connect our cast of characters. The key tool is the Bernstein-Gelfand-Gelfand correspondence, which is an isomorphism between the category of linear free complexes over the exterior algebra $E=\Lambda(V)$ and the category of graded free modules over the symmetric algebra $S=\operatorname{Sym}\left(V^{*}\right)$. An introduction to the BGG correspondence may be found in Chapter 7 of 12 ; additional sources are [13, 9], and [14.

Notice that if we take $\operatorname{Sym}\left(V^{*}\right)$ to be generated in degree one, then $\bigwedge(V)$ is generated in degree -1 . The convention in arrangement theory (and the convention of this paper) is that the exterior algebra is generated in degree 1. To distinguish between gradings, we write $E^{\prime}$ for an exterior algebra with generators in degree -1 , and $E$ if the generators are in degree 1.

Let $\mathbf{L}$ denote the functor from the category of graded $E^{\prime}$-modules to the category of linear free complexes over $S$, defined as follows: for a graded $E^{\prime}$-module $P, \mathbf{L}(P)$ is the complex

$$
\cdots \longrightarrow P_{i} \otimes S \stackrel{d_{i}}{\longrightarrow} P_{i-1} \otimes S \longrightarrow \cdots
$$

with differentials $d_{i}: p \otimes 1 \rightarrow \sum e_{i} p \otimes x_{i}$. We will be applying the functor $\mathbf{L}$ to the $E^{\prime}$-module $A^{\prime}=E^{\prime} / I \otimes E^{\prime}(-\ell)$, where $I$ is the Orlik-Solomon ideal; tensoring with $E^{\prime}(-\ell)$ shifts so the unit of the algebra is in degree $\ell$, and the generators are in degree $\ell-1$, with $\ell$ the dimension of the ambient space of the arrangement.

Similarly, let $\mathbf{R}$ denote the functor from the category of graded $S$-modules to the category of linear free complexes over $E^{\prime}$ : for a graded $S$-module $M, \mathbf{R}(M)$ is the complex

$$
\cdots \longrightarrow \operatorname{Hom}_{\mathbb{k}}\left(E^{\prime}, M_{i}\right) \longrightarrow \operatorname{Hom}_{\mathbb{k}}\left(E^{\prime}, M_{i+1}\right) \longrightarrow \cdots
$$

In Theorem 4.3 of [13, Eisenbud, Fløystad and Schreyer show that if $M$ is a graded $S$-module, with linear free resolution given by $\mathbf{L}(P)$, then the dimension of $\operatorname{Tor}_{i}^{E^{\prime}}(P, \mathbb{k})$ can be computed from the dimensions of the graded pieces of the local cohomology modules of $M$. This result will be the main tool in our application of BGG; for completeness (and because of the grading differences) we prove a variant of their result in $\$ 3.3$ below.

3.2. The Eisenbud-Popescu-Yuzvinsky resolution. Let $\mathcal{A}=\left\{H_{1}, \ldots, H_{n}\right\}$ be a central arrangement in $\mathbb{C}^{\ell}$, with complement $X(\mathcal{A})$. Identify the cohomology ring $H^{*}(X(\mathcal{A}), \mathbb{k})$ with the Orlik-Solomon algebra $A=E / I$, where $E$ is the exterior algebra on $V=\mathbb{k}^{n}$. The BGG correspondence was used in this context by Eisenbud, Popescu and Yuzvinsky [14 to establish that $H_{*}(X(\mathcal{A}), \mathbb{k}) \cong \operatorname{ann}(I)$ has a linear free resolution over $E$; results on the first differentials in this resolution appear in [10.

Fix a basis $e_{1}, \ldots, e_{n}$ for $V$, and let $x_{1}, \ldots, x_{n}$ be the dual basis for $V^{*}$. From 14], Corollary 3.2, we have an exact sequence of $S$-modules:

$$
0 \longrightarrow A_{0} \otimes S \stackrel{d_{0}}{\longrightarrow} A_{1} \otimes S \stackrel{d_{1}}{\longrightarrow} \cdots \stackrel{d_{\ell-1}}{\longrightarrow} A_{\ell} \otimes S \longrightarrow F(A) \longrightarrow 0 .
$$

The key point here is that the complex obtained by applying BGG to $A$ is in fact exact, hence a free resolution of $F(A)$. Notice that the differential $d_{i}: p \otimes 1 \mapsto$ $\sum_{i=1}^{n} e_{i} p \otimes x_{i}$ is precisely the differential in the Aomoto complex $(A, a)$, where the 
maps are given by multiplication by a generic linear form of the exterior algebra; the $x_{i}$ are simply the coefficients of this form. With grading convention that $E$ is generated in degree one, $F(A)$ is generated in degree $-\ell$. With this choice, $\operatorname{Ext}_{S}^{\ell-1}(F(A), S)$ is generated in degree $\ell-1$; we will see in a bit that this is consistent with the topological formula (2.7).

3.3. Relating $\operatorname{Tor}^{E}$ and $\operatorname{Ext}_{S}$. The following lemma is a restatement of Theorem 4.3 of [13]. For our purposes it is preferable to use $\operatorname{Ext}^{i}(\bullet, S)$ rather than the local cohomology modules $H_{\mathfrak{m}}^{i}(\bullet)$; by local duality (11], Theorem A.4.2) these modules encode essentially the same information.

Lemma 3.1. With notation as above, we have

$$
\operatorname{dim}_{\mathbb{k}} \operatorname{Tor}_{i}^{E}(A, \mathbb{k})_{j}=\operatorname{dim}_{\mathbb{k}} \operatorname{Ext}_{S}^{i+\ell-j}(F(A), S)_{j} .
$$

Proof. As before, write $A^{\prime}$ for the Orlik-Solomon algebra, with unit in degree $\ell$, and generators in degree $\ell-1$. A straightforward translation shows that

$$
\operatorname{dim}_{\mathbb{k}} \operatorname{Tor}_{i}^{E}(A, \mathbb{k})_{j}=\operatorname{dim}_{\mathbb{k}} \operatorname{Tor}_{i}^{E^{\prime}}\left(A^{\prime}, \mathbb{k}\right)_{\ell-j} .
$$

Let $C$ denote the Cartan complex; $C$ is the minimal free resolution of $\mathbb{k}$ as an $E^{\prime}$-module. For any $E^{\prime}$-module $P$,

$$
\operatorname{Tor}_{i}^{E^{\prime}}(P, \mathbb{k})=H_{i}(P \otimes C) .
$$

By Proposition 7.9 and Exercise 7.7 of [12], $H_{k}\left(\operatorname{Hom}_{S}(\mathbf{L}(P), S)\right)_{i+k}$ is dual to $H_{i}(P \otimes C)_{-i-k}$. If we apply this with $P=A^{\prime}$, then [14 tells us that $\mathbf{L}\left(A^{\prime}\right)$ is a free resolution of $F\left(A^{\prime}\right)$, so that ([11, A3.11)

$$
H_{k}\left(\operatorname{Hom}_{S}\left(\mathbf{L}\left(A^{\prime}\right), S\right)\right)=\operatorname{Ext}^{-k}\left(F\left(A^{\prime}\right), S\right) .
$$

The reason for tensoring so that $A^{\prime}$ has no components of negative degree is that it keeps the indexing simple; in particular, we find that

$$
\operatorname{dim}_{\mathbb{k}} \operatorname{Tor}_{i}^{E^{\prime}}\left(A^{\prime}, \mathbb{k}\right)_{\ell-j}=\operatorname{dim}_{\mathbb{k}} \operatorname{Ext}_{S}^{i+\ell-j}\left(F\left(A^{\prime}\right), S\right)_{j-\ell} .
$$

Finally, since $F\left(A^{\prime}\right)$ is generated in degree zero, $F(A)=F\left(A^{\prime}\right) \otimes S(\ell)$ is generated in degree $-\ell$, as claimed. So

$$
\begin{aligned}
\operatorname{dim}_{\mathbb{k}} \operatorname{Hom}_{S}(F(A), S)_{t} & =\operatorname{dim}_{\mathbb{k}} \operatorname{Hom}_{S}\left(F\left(A^{\prime}\right) \otimes S(\ell), S\right)_{t} \\
& =\operatorname{dim}_{\mathbb{k}} \operatorname{Hom}_{S}\left(F\left(A^{\prime}\right), S\right)_{t-\ell .}
\end{aligned}
$$

Thus,

$$
\operatorname{dim}_{\mathbb{k}} \operatorname{Ext}_{S}^{i+\ell-j}\left(F\left(A^{\prime}\right), S\right)_{j-\ell}=\operatorname{dim}_{\mathbb{k}} \operatorname{Ext}_{S}^{i+\ell-j}(F(A), S)_{j} .
$$

Putting together (3.4), (3.7), and (3.9) finishes the proof.

Recall that the regularity of an $E$-module $M$ is the smallest integer $n$ such that $\operatorname{Tor}_{i}^{E}(M, \mathbb{k})_{j}=0$ for all $i, j$ with $j \geq i+n+1$. Lemma 2.3 of [30] is that $A$ is $(\ell-1)$ regular; this also follows from Lemma 3.1 and the EPY resolution of $F(A)$. For example, if we write $e_{i j}$ for $\operatorname{dim}_{\mathbb{k}} \operatorname{Ext}_{S}^{i}(F(A), S)_{j}$, then the minimal free resolution of $A$ over $E$ (for $\ell=3$ ) is

$$
0 \longleftarrow A \longleftarrow E \longleftarrow E^{e_{22}}(-2) \oplus E^{e_{13}}(-3) \longleftarrow E^{e_{23}}(-3) \oplus E^{e_{14}}(-4) \longleftarrow \cdots .
$$


3.4. The module $\mathfrak{B}$ as an Ext module. We now connect the players. Recall that the linearized Alexander invariant (over $\mathbb{k}$ ) is the $S$-module defined via the exact sequence

$$
\left(A_{2} \oplus E_{3}\right) \otimes S \stackrel{\alpha_{2} \otimes \mathrm{id}+\delta_{3}}{\longrightarrow} E_{2} \otimes S \longrightarrow \mathfrak{B} \longrightarrow 0,
$$

where $\alpha_{k}: A_{k} \rightarrow E_{k}$ is the adjoint of the canonical surjection $\gamma_{k}: E_{k} \rightarrow A_{k}$ (recall, we write $\mathfrak{B}$ instead of $\mathfrak{B} \otimes \mathbb{k}$, for simplicity).

Theorem 3.2. The linearized Alexander invariant is functorially determined by the Orlik-Solomon algebra, as follows:

$$
\mathfrak{B} \cong \operatorname{Ext}_{S}^{\ell-1}(F(A), S) .
$$

Proof. Consider the following commuting diagram, with exact rows and columns:

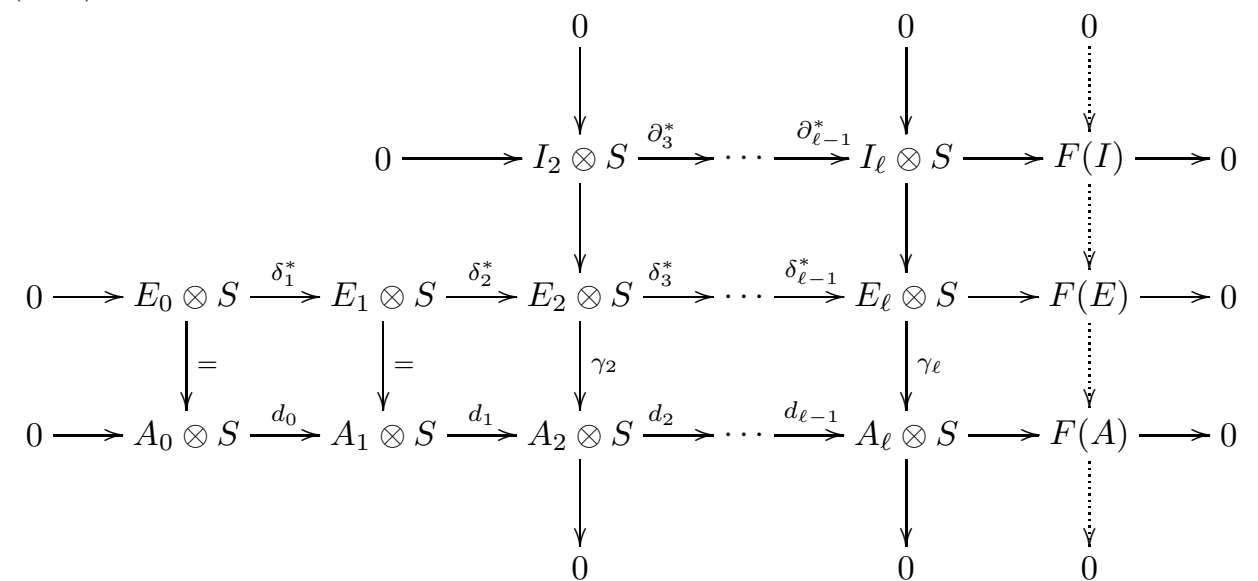

The rows in the diagram are EPY resolutions. Notice that the middle row is just a truncation of the Koszul complex over $S$. All columns but the last one (with solid arrows) are exact by definition of the Orlik-Solomon algebra $A=E / I$. The exactness of the two bottom rows, combined with the long exact sequence in homology, shows that the rightmost column (marked with dotted arrows) is exact. This short exact sequence yields a long exact sequence of Ext modules:

$$
\cdots \longrightarrow \operatorname{Ext}_{S}^{i}(F(E), S) \longrightarrow \operatorname{Ext}_{S}^{i}(F(I), S) \longrightarrow \operatorname{Ext}_{S}^{i+1}(F(A), S) \longrightarrow \cdots .
$$

The Koszul complex is exact and self-dual, so $\operatorname{Ext}_{S}^{i}(F(E), S)=0$, for all $i<\ell$, thus $\operatorname{Ext}_{S}^{i+1}(F(A), S) \cong \operatorname{Ext}_{S}^{i}(F(I), S)$, for $i<\ell-1$. Since all the vertical exact sequences (except the last one) consist of free $S$-modules, the dual sequences are also exact, and so $E_{k} /\left(\operatorname{im} \alpha_{k}\right) \cong\left(I_{k} \otimes S\right)^{*}$. Hence,

$$
\begin{aligned}
\mathfrak{B} & =E_{2} \otimes S / \operatorname{im}\left(\alpha_{2} \otimes \mathrm{id}+\delta_{3}\right) \\
& \cong\left(I_{2} \otimes S\right)^{*} / \operatorname{im}\left(\partial_{3}\right) \\
& \cong \operatorname{Ext}_{S}^{\ell-2}(F(I), S) \\
& \cong \operatorname{Ext}_{S}^{\ell-1}(F(A), S) .
\end{aligned}
$$

If char $\mathbb{k}=0$ (which recall is our standing assumption), then we obtain the following corollary. 
Corollary 3.3. The Chen ranks of an arrangement group $G$ equal the graded Betti numbers of the linear strand of the cohomology ring $A$ over the exterior algebra $E$ :

$$
\theta_{k}(G)=\operatorname{dim}_{\mathbb{k}} \operatorname{Tor}_{k-1}^{E}(A, \mathbb{k})_{k}, \quad \text { for all } k \geq 2 .
$$

Proof. We have

$$
\begin{aligned}
\theta_{k}(G) & =\operatorname{dim}_{\mathbb{k}} \mathfrak{B}_{k} & & \text { (by Theorem 2.2) } \\
& =\operatorname{dim}_{\mathbb{k}} \operatorname{Ext}_{S}^{\ell-1}(F(A), S)_{k} & & \text { (by Theorem 3.2) } \\
& =\operatorname{dim}_{\mathbb{k}} \operatorname{Tor}_{k-1}^{E}(A, \mathbb{k})_{k} & & \text { (by Lemma 3.1). }
\end{aligned}
$$

In particular, this shows that Conjectures $\mathrm{A}$ and $\mathrm{B}$ and are equivalent. We note that it is possible to assemble a different proof of Corollary 3.3 using recent results of Fröberg and Löfwall [19] on Koszul homology and homotopy Lie algebras.

3.5. Chen groups of graphic arrangements. We conclude this section with an application to a particularly nice class of arrangements. Given a simple graph $\Gamma$, with vertex set $\mathcal{V}=\{1, \ldots, n\}$ and edge set $\mathcal{E}$, the corresponding graphic arrangement, $\mathcal{A}(\Gamma)=\left\{H_{e}\right\}_{e \in \mathcal{E}}$, consists of the hyperplanes in $\mathbb{C}^{n}$ of the form $H_{e}=\left\{z \in \mathbb{C}^{n} \mid z_{i}-z_{j}=0\right\}$ for which $e=(i, j)$ belongs to $\mathcal{E}$.

For example, if $\Gamma=K_{n}$ is the complete graph on $n$ vertices, then $\mathcal{A}\left(K_{n}\right)$ is the braid arrangement in $\mathbb{C}^{n}$. The resonance components of $\mathcal{A}\left(K_{n}\right)$ are in one-to-one correspondence to the 3 -vertex and 4 -vertex subsets of $\mathcal{V}$, and are all 1-dimensional; see [8, §6.8], and compare with Example 2.3.

Now, since every graph on $n$ vertices is a subgraph of the complete graph $K_{n}$, every graphic arrangement is a sub-arrangement of the braid arrangement $\mathcal{A}\left(K_{n}\right)$. It follows that $\mathcal{R}^{1}(\mathcal{A}(\Gamma))$ has precisely one, 1-dimensional component for each triangle or complete quadrangle in $\Gamma$.

Combining Corollary 3.3 with our previous results from [30, where the linear strand of a graphic arrangement is determined, we see that the Chen ranks conjecture holds for graphic arrangements. More explicitly, we have the following.

Theorem 3.4. Let $\Gamma$ be a graph, $\mathcal{A}=\mathcal{A}(\Gamma)$ the corresponding graphic arrangement, and $G=\pi_{1}(X(\mathcal{A}))$ its group. Then

$$
\theta_{k}(G)=(k-1)\left(\kappa_{2}+\kappa_{3}\right), \quad \text { for all } k \geq 3,
$$

where $\kappa_{s}$ denotes the number of complete subgraphs on $s+1$ vertices.

Example 3.5. If $\mathcal{A}=\mathcal{A}\left(K_{n}\right)$ is the braid arrangement in $\mathbb{C}^{n}$, then $G$ is isomorphic to $P_{n}$, the pure braid group on $n$ strings. Applying formula (3.13), we find that

$$
\begin{aligned}
\theta_{k}\left(P_{n}\right) & =(k-1) \cdot\left(\left(\begin{array}{l}
n \\
3
\end{array}\right)+\left(\begin{array}{l}
n \\
4
\end{array}\right)\right) \\
& =(k-1) \cdot\left(\begin{array}{c}
n+1 \\
4
\end{array}\right), \quad \text { for all } k \geq 3 .
\end{aligned}
$$

The Chen ranks of the pure braid groups were first computed in [6], using an arduous Gröbner basis computation. The above computation recovers the result of [6]. 


\section{The rate of growth of the Chen groups}

It follows from Theorem 2.2 that for $k \gg 0$ the Chen rank $\theta_{k}$ is given by a rational polynomial $\mathrm{P}(k)$. In this section, we prove that the degree of $\mathrm{P}(k)$ is equal to the dimension of $\mathcal{R}^{1}(\mathcal{A})$. As an application, we compute the complexity of the Orlik-Solomon algebra $A$, viewed as a module over the exterior algebra $E$, in the case when $\mathcal{A}$ is a central arrangement in $\mathbb{C}^{3}$.

4.1. Primary decomposition of $\operatorname{ann}(\mathfrak{B})$. We know that the Fitting ideal of the presentation matrix of $\mathfrak{B}$ has the same radical as the annihilator of $\mathfrak{B}$, and by [8], the corresponding variety is precisely $\mathcal{R}^{1}(\mathcal{A})$. This variety has a number of nice properties: as noted earlier, Libgober and Yuzvinsky 22] showed that it is the union of disjoint projective subspaces, each of dimension at least one.

For each component $L \subset \mathcal{R}^{1}(\mathcal{A})$, denote by $\mathfrak{p}=\mathfrak{p}_{L}$ the corresponding minimal prime ideal in $S$. Slightly abusing notation, we write $\operatorname{Ass}\left(\mathcal{R}^{1}(\mathcal{A})\right)$ for the set of such ideals. For each $\mathfrak{p} \in \operatorname{Ass}\left(\mathcal{R}^{1}(\mathcal{A})\right)$, denote by $L=L_{\mathfrak{p}}$ the corresponding linear subspace of $\mathcal{R}^{1}(\mathcal{A})$. Then

$$
\mathcal{R}^{1}(\mathcal{A})=\bigcup_{\mathfrak{p} \in \operatorname{Ass}\left(\mathcal{R}^{1}(\mathcal{A})\right)} L_{\mathfrak{p}} \quad \text { and } \quad \sqrt{\operatorname{ann}(\mathfrak{B})}=\bigcap_{\mathfrak{p} \in \operatorname{Ass}\left(\mathcal{R}^{1}(\mathcal{A})\right)} \mathfrak{p} .
$$

For each $\mathfrak{p} \in \operatorname{Ass}\left(\mathcal{R}^{1}(\mathcal{A})\right)$, there is at least one homogeneous element $m \in \mathfrak{B}$ whose annihilator is $\mathfrak{p}$, i.e., $\langle m\rangle \cong S / \mathfrak{p}$, as $S$-modules. Let $M(\mathfrak{p})$ denote the submodule of $\mathfrak{B}$ annihilated by $\mathfrak{p}$ :

$$
M(\mathfrak{p})=\{x \in \mathfrak{B} \mid \operatorname{ann}(x)=\mathfrak{p}\} .
$$

It is easy to see that there is almost no interaction between these (finitely-generated) submodules.

Lemma 4.1. Let $N$ be a finitely-generated, graded $S$-module, and $n_{1}, n_{2} \in N$ satisfy $n_{1} \notin\left\langle n_{2}\right\rangle$ and $n_{2} \notin\left\langle n_{1}\right\rangle$. Suppose ann $\left\langle n_{i}\right\rangle=P_{i} \subsetneq \mathfrak{m}$ and $P_{1}+P_{2}=\mathfrak{m}$, where $\mathfrak{m}$ is the maximal homogeneous ideal. Then

$$
\left\langle n_{1}\right\rangle \cap\left\langle n_{2}\right\rangle=0
$$

Proof. Suppose $x=a_{1} n_{1}=a_{2} n_{2}$, with $a_{i} \in S$. Then obviously both $P_{1}$ and $P_{2}$ annihilate $x$, so the maximal ideal $\mathfrak{m}$ kills $x$. By degree considerations, we must have $a_{i} \in \mathfrak{m}$. Since $\mathfrak{m} \cdot a_{1} n_{1}=0$, we find that $a_{1}^{2} n_{1}=0$. But $P_{1}$ is prime, so $a_{1}^{2} \in P_{1}$ implies $a_{1} \in P_{1}$. Hence $x=0$.

4.2. Local cohomology and regularity. Associated to any finitely generated, graded module $N$, there is an exact sequence (see Eisenbud [11]),

$$
0 \longrightarrow H_{\mathfrak{m}}^{0}(N) \longrightarrow N \longrightarrow \sum_{v \in \mathbb{Z}} H^{0}(\tilde{N}(v)) \longrightarrow H_{\mathfrak{m}}^{1}(N) \longrightarrow 0
$$

where $H_{\mathfrak{m}}^{*}(N)$ denotes the local cohomology of $N$, supported at the maximal ideal $\mathfrak{m}$, and $\widetilde{N}$ is the sheaf associated to $N$.

In [33. Yuzvinsky showed that the module $F(A)$ is annihilated by $f=\sum_{i=1}^{n} x_{i}$; thus $F(A)_{f}=0$. Localization commutes with the Ext functor, so for any $i$, $\operatorname{Ext}_{S}^{i}(F(A), S)_{f}=0$, and we conclude that $f$ annihilates $\mathfrak{B}=\operatorname{Ext}_{S}^{\ell-1}(F(A), S)$. In particular, every element of $\mathfrak{B}$ is torsion, hence every element of $\mathfrak{B}$ belongs to 
some $M(\mathfrak{p})$. Combined with Lemma 4.1 this shows that the sheaf associated to $\mathfrak{B}$ decomposes as a direct sum of the sheaves associated to the modules $M(\mathfrak{p})$ :

$$
\widetilde{\mathfrak{B}} \cong \bigoplus_{\mathfrak{p} \in \operatorname{Ass}\left(\mathcal{R}^{1}(\mathcal{A})\right)} \widetilde{M}(\mathfrak{p})
$$

The modules $H_{\mathfrak{m}}^{0}(\mathfrak{B})$ and $H_{\mathfrak{m}}^{1}(\mathfrak{B})$ vanish in high degree (see Chapter 9 of [12]), so the exact sequence above yields a coarse approximation to the Chen ranks conjecture: for $k>\operatorname{reg}(\mathfrak{B})$,

$$
\theta_{k}(G)=\mathrm{P}(\mathfrak{B}, k)=\sum_{p \in \operatorname{Ass}\left(\mathcal{R}^{1}(\mathcal{A})\right)} \mathrm{P}(M(\mathfrak{p}), k) .
$$

Lemma 4.2. Let $\mathfrak{p}$ be a prime ideal in $\operatorname{Ass}\left(\mathcal{R}^{1}(\mathcal{A})\right)$. Then $\operatorname{deg} \mathrm{P}(M(\mathfrak{p}), k)=$ $\operatorname{dim} L_{\mathfrak{p}}$.

Proof. The module $M(\mathfrak{p})$ is generated by finitely many homogeneous elements, each having annihilator $\mathfrak{p}$. Let $m$ be one of those elements, and $\langle m\rangle$ the submodule it generates. Then $\langle m\rangle \cong S(-d) / \mathfrak{p}$, where $d$ is the degree of $m$. On the other hand, $L_{\mathfrak{p}}=V(\mathfrak{p})$ is a linear subspace of $E_{1}$, say of dimension $r$. Hence,

$$
\mathrm{P}(\langle m\rangle, k)=\mathrm{P}(S(-d) / \mathfrak{p}, k)=\left(\begin{array}{c}
r+k-d \\
k-d
\end{array}\right)=\frac{k^{r}}{r !}+\cdots,
$$

and so $\operatorname{deg} \mathrm{P}(\langle m\rangle, k)=r$. Since $\langle m\rangle$ is a submodule of $M(\mathfrak{p})$, this implies

$$
\operatorname{deg} \mathrm{P}(M(\mathfrak{p}), k) \geq r .
$$

On the other hand, $M(\mathfrak{p})$ is generated by finitely many such submodules, and so (4.7) also implies $\operatorname{deg} \mathrm{P}(M(\mathfrak{p}), k) \leq r$. ing.

Combining Theorem 2.2. Equation (4.5), and Lemma 4.2, we obtain the follow-

Theorem 4.3. There exists a polynomial $\mathrm{P}(t) \in \mathbb{Q}[t]$, of degree equal to the dimension of the projective variety $\mathcal{R}^{1}(\mathcal{A})$, such that

$$
\theta_{k}(G)=\mathrm{P}(k), \quad \text { for all } k>\operatorname{reg}(\mathfrak{B}) .
$$

4.3. The complexity of the OS-algebra. Let $M$ be a finitely generated module over a local or graded ring $R$ with residue field $\mathbb{k}$, and let

$$
\beta_{i}(M)=\operatorname{dim}_{\mathbb{k}} \operatorname{Tor}_{i}^{R}(M, \mathbb{k})
$$

be the rank of the $i$ th free module in a minimal free resolution of $M$ over $R$. We then have the following measure of the growth of the numbers $\beta_{i}(M)$; see 3 .

Definition 4.4. The complexity of the module $M$ is the integer

$$
\operatorname{cx}_{R}(M)=\inf \left\{d \in \mathbb{Z} \mid \exists f(t) \in \mathbb{R}[t] \text { of degree } d-1 \text {, with } \beta_{i}(M) \leq f(i), \forall i \geq 1\right\} .
$$

Now let $\mathcal{A}$ be a central arrangement of $n$ planes in $\mathbb{C}^{3}$. We wish to compute the complexity of the Orlik-Solomon algebra $A=E / I$, viewed as a module over $E$. We start with an observation about the resonance variety $\mathcal{R}^{1}(\mathcal{A})$.

Lemma 4.5. If $\mathcal{A}$ is not a pencil, then $\operatorname{dim} \mathcal{R}^{1}(\mathcal{A})<n-2$. 
Proof. If $\operatorname{dim} \mathcal{R}^{1}(\mathcal{A}) \geq n-2$, then since $\mathcal{R}^{1}(\mathcal{A})$ is the union of a subspace arrangement in $\mathbb{P}^{n-1}$, it must contain a hyperplane $H \cong \mathbb{P}^{n-2}$. But since the components of $\mathcal{R}^{1}(\mathcal{A})$ are linear subspaces of dimension at least one, Bézout's theorem says any component $L$ of $\mathcal{R}^{1}(\mathcal{A})$ different from $H$ must meet $H$, contradicting the fact that $\mathcal{R}^{1}(\mathcal{A})$ consists of disjoint subspaces (notice that applying Bézout makes sense, because linear subspaces are disjoint over $\mathbb{k}$ iff they are disjoint over $\bar{k}$ ). Thus $\mathcal{R}^{1}(\mathcal{A})=H$, and so $\mathcal{R}^{1}(\mathcal{A})$ contains a single component (necessarily local), of dimension $n-2$. Hence $\mathcal{A}$ is a pencil.

Write $b_{i, j}^{\prime}=\operatorname{dim}_{\mathbb{k}} \operatorname{Tor}_{i}^{E}(A, \mathbb{k})_{j}$. We noted in [30] that the regularity of $A$ as an $E$-module is two; so $\beta_{i}(A)=b_{i, i+1}^{\prime}+b_{i, i+2}^{\prime}$. Using the (minimal, free) resolution of $A$ over $E$, we can then write the Hilbert series of $A$ as

$$
\sum_{i=0}^{3} b_{i} t^{i}=(1+t)^{n} \sum_{i \geq 0}(-1)^{i}\left(b_{i, i+1}^{\prime} t^{i+1}+b_{i, i+2}^{\prime} t^{i+2}\right) .
$$

Since $b_{0}=1, b_{1}=n$, and $b_{3}=b_{2}-n+1$ (see [27]), we have

$$
\frac{1+n t+b_{2} t^{2}+\left(b_{2}-n+1\right) t^{3}}{(1+t)^{n}}=\sum_{i \geq 0}(-1)^{i}\left(b_{i, i+1}^{\prime} t^{i+1}+b_{i, i+2}^{\prime} t^{i+2}\right) .
$$

Expanding the left-hand side of (4.10), we find it equals

$$
1+\sum_{m=2}^{\infty}(-1)^{m}\left(\begin{array}{c}
n+m-4 \\
m-2
\end{array}\right) \cdot \frac{3(m-2)+m b_{2}-(2 m-5) n-n^{2}}{m} t^{m} .
$$

Assume $m$ is odd, and expand the coefficient of $t^{m}$ as a polynomial in $m$. We find that it is equal to $\frac{-b_{2}+2 n-3}{(n-2) !} m^{n-2}+\cdots$. On the other hand, the coefficient of $t^{m}$ on the right-hand side of (4.10) is $b_{m-1, m}^{\prime}-b_{m-2, m}^{\prime}$. Now recall from (3.12) that $\theta_{k}=b_{k-1, k}^{\prime}$, for all $k \geq 2$. Thus, we obtain

$$
b_{m-2, m}^{\prime}=\theta_{m-1}+\frac{b_{2}-2 n+3}{(n-2) !} m^{n-2}+\cdots .
$$

A similar argument applies when $m$ is even. Notice that $b_{2}-2 n+3=0$ iff $\mathcal{A}$ is a near pencil, i.e., $n-1$ planes through a line, and one additional plane in general position.

Proposition 4.6. If $\mathcal{A}$ is a central arrangement of $n$ planes in $\mathbb{C}^{3}$, and $\mathcal{A}$ is not a near pencil, then $\operatorname{cx}_{E}(A)=n-1$. If $\mathcal{A}$ is a near pencil, then $\operatorname{cx}_{E}(A)=n-2$.

Proof. If $\mathcal{A}$ is a near pencil, then the resolution of $A$ over $E$ is linear, and we know that $\beta_{m}(A)=\theta_{m}$ is a polynomial in $m$ of degree $n-3$. If $\mathcal{A}$ is a pencil, then the resolution is also linear, and $\beta_{m}(A)=\theta_{m}$ is a polynomial of degree $n-2$. If $\mathcal{A}$ is not a pencil or near pencil, then by Lemma 4.5, $\operatorname{dim} \mathcal{R}^{1}(\mathcal{A})<n-2$. It follows from Theorem 4.3 that $\theta_{m}$ is a polynomial of degree at most $n-3$, so $\beta_{m}(A)$ is a polynomial of degree $n-2$, for $m \gg 0$.

Remark 4.7. In Theorem 3.1 of [2], Aramova, Avramov, and Herzog show that the complexity of an $E$-module is equal to the dimension of the (affine) rank variety; in Corollary 2.3 of [14, Eisenbud, Popescu, and Yuzvinsky prove that when the module in question is the Orlik-Solomon algebra, the codimension of the rank variety is equal to the number of central factors in an irreducible decomposition of $\mathcal{A}$. On 
the other hand, near pencils are the only reducible arrangements in rank $\ell=3$. So Proposition 4.6 is in fact an instance of a more general result.

\section{A LOWER BOUND FOR THE CHEN RANKS}

In this section we prove a lower bound on the Chen ranks of an arrangement group. We continue to use the notation of the previous section.

The idea of the proof is as follows. First, we choose an irreducible component of $\mathcal{R}^{1}(\mathcal{A})$ (we do not distinguish between the local and non-local components), which corresponds to a sub-ideal of $I_{2}$, generated by decomposable elements. A short exact sequence relates this sub-ideal of decomposable elements to $I$. After appealing to the Bernstein-Gelfand-Gelfand correspondence and dualizing, we obtain a long exact sequence of Ext-modules. Finally, knowledge of the geometry of $\mathcal{R}^{1}(\mathcal{A})$, combined with a localization argument, yields the bound.

5.1. The modules $\mathfrak{B}(\mathfrak{p})$. Let $\mathfrak{p} \in \operatorname{Ass}\left(\mathcal{R}^{1}(\mathcal{A})\right)$ be a minimal prime ideal, and $L_{\mathfrak{p}}=V(\mathfrak{p}) \subset E_{1}$ the corresponding linear subspace. As shown by Falk in 16, Corollary 3.11, the following holds: If $a, b \in L_{\mathfrak{p}}$, then $a \wedge b \in I_{2}$. Thus, if we define

$$
I(\mathfrak{p})=\left\langle a \wedge b \mid a, b \in L_{\mathfrak{p}}\right\rangle
$$

to be the ideal of $E$ generated by wedge products of pairs of elements from $L_{\mathfrak{p}}$, then $I(\mathfrak{p})$ is a sub-ideal of $I$.

Recall from Theorem 3.2 that $\mathfrak{B} \cong \operatorname{Ext}_{S}^{\ell-1}(F(A), S)$. By analogy, define the linearized Alexander invariant at $\mathfrak{p}$ to be the $S$-module

$$
\mathfrak{B}(\mathfrak{p})=\operatorname{Ext}_{S}^{\ell-2}(F(I(\mathfrak{p})), S) .
$$

Example 5.1. Let $\mathcal{A}=\left\{H_{0}, \ldots, H_{n}\right\}$ be a pencil of lines in $\mathbb{C}^{2}$. Let $E$ be the exterior algebra on $e_{0}, \ldots, e_{n}$. The Orlik-Solomon ideal $I$ is generated by $\left\{\partial e_{0 i j}\right\}$, while the resonance variety $\mathcal{R}^{1}(\mathcal{A})$ consists of a single component $L_{\mathfrak{p}}=\operatorname{span}\left\{e_{0}-e_{i}\right\}$, with $\mathfrak{p}=\left\langle\sum_{i=0}^{n} x_{i}, x_{r+1}, \ldots, x_{n}\right\rangle$ and $I(\mathfrak{p})=I$. After a linear change of variables in $E$, the ideal $I$ corresponds to $I_{0}=\left\langle e_{1}, \ldots, e_{n}\right\rangle^{2}$, while the linear subspace $L_{\mathfrak{p}}$ corresponds to $L_{0}=\operatorname{span}\left\{e_{1}, \ldots, e_{n}\right\}$. A standard calculation (compare [7]) shows that the module $\mathfrak{B}(\mathfrak{p})$ has Hilbert polynomial $\mathrm{P}(\mathfrak{B}(\mathfrak{p}), k)=(k-1)\left(\begin{array}{c}n+k-2 \\ k\end{array}\right)$.

Example 5.2. Let $\mathcal{A}$ be the braid arrangement, discussed in Example 2.3. The generators of $I_{2}$ are $f_{1}=\partial e_{145}, f_{2}=\partial e_{235}, f_{3}=\partial e_{034}$, and $f_{4}=\partial e_{012}$. These give rise to various "local" data. For example, $\mathfrak{p}_{1}=\left\langle x_{0}, x_{2}, x_{3}, \sum x_{i}\right\rangle, L_{\mathfrak{p}_{1}}=$ $\operatorname{span}\left\{e_{1}-e_{4}, e_{1}-e_{5}\right\}, I\left(\mathfrak{p}_{1}\right)=\left\langle\left(e_{1}-e_{4}\right)\left(e_{1}-e_{5}\right)\right\rangle$, and $\mathfrak{B}\left(\mathfrak{p}_{1}\right)=\operatorname{coker}\left(\vartheta_{\mathfrak{p}_{1}}: S^{4} \rightarrow S\right)$, where $\vartheta_{\mathfrak{p}_{1}}=\left(\begin{array}{llll}x_{0} & x_{2} & x_{3} & \sum x_{i}\end{array}\right)$.

Now recall $\mathcal{A}$ supports a non-trivial neighborly partition, $\Pi=(05|13| 24)$. The corresponding non-local component $L_{\Pi} \subset \mathcal{R}^{1}(\mathcal{A})$ is spanned by $\eta_{1}=e_{0}-e_{1}-e_{3}+$ $e_{5}$ and $\eta_{2}=e_{0}-e_{2}-e_{4}+e_{5}$. The associated prime is $\mathfrak{p}=\left\langle x_{0}-x_{5}, x_{1}-x_{3}\right.$, $\left.x_{2}-x_{4}, \sum x_{i}\right\rangle$, whereas $I(\mathfrak{p})=\left\langle\eta_{1} \wedge \eta_{2}\right\rangle$. Finally, $\mathfrak{B}(\mathfrak{p})=\operatorname{coker}\left(\vartheta_{\mathfrak{p}}: S^{4} \rightarrow S\right)$, where $\vartheta_{\mathfrak{p}}=\left(\begin{array}{llll}x_{0}-x_{5} & x_{1}-x_{3} & x_{2}-x_{4} & \sum x_{i}\end{array}\right)$.

Lemma 5.3. If $\operatorname{dim} L_{\mathfrak{p}}=\operatorname{dim} L_{\mathfrak{q}}$, there is a linear automorphism of $E$ taking $I(\mathfrak{p})$ to $I(\mathfrak{q})$.

Proof. After a suitable linear change of variables, we can assume $L_{\mathfrak{p}}$ is the subspace spanned by $\left\{e_{1}, \ldots, e_{r}\right\}$, where $r=\operatorname{dim} L_{\mathfrak{p}}$. Let $I(\mathfrak{p})$ be the corresponding ideal of $E$. We know that every element $e_{i} \wedge e_{j}(1 \leq i<j \leq r)$ is in $I(\mathfrak{p})$. Hence, $I(\mathfrak{p})=\left\langle e_{1}, \ldots, e_{r}\right\rangle^{2}$. 
In particular, the sub-ideals $I(\mathfrak{p})$ (and thus, the modules $B(\mathfrak{p})$ ) do not distinguish between local and non-local resonance. In view of Example 5.1, we obtain the following.

Corollary 5.4. The Hilbert polynomial of $\mathfrak{B}(\mathfrak{p})$ is given by

$$
\mathrm{P}(\mathfrak{B}(\mathfrak{p}), k)=(k-1)\left(\begin{array}{c}
\operatorname{dim} L_{\mathfrak{p}}+k-1 \\
k
\end{array}\right) .
$$

5.2. Snake lemma and localization. For simplicity, we will assume from now on that $\mathcal{A}$ is a central arrangement in $\mathbb{C}^{3}$. For a fixed $\mathfrak{p} \in \operatorname{Ass}\left(\mathcal{R}^{1}(\mathcal{A})\right)$, consider the short exact sequence of $E$-modules

$$
0 \longrightarrow I(\mathfrak{p}) \longrightarrow I \longrightarrow I / I(\mathfrak{p}) \longrightarrow 0 \text {. }
$$

Using $I_{1}=0$ (which also implies $I(\mathfrak{p})_{1}=0$ ), we obtain the following commuting diagram of $S$-modules:

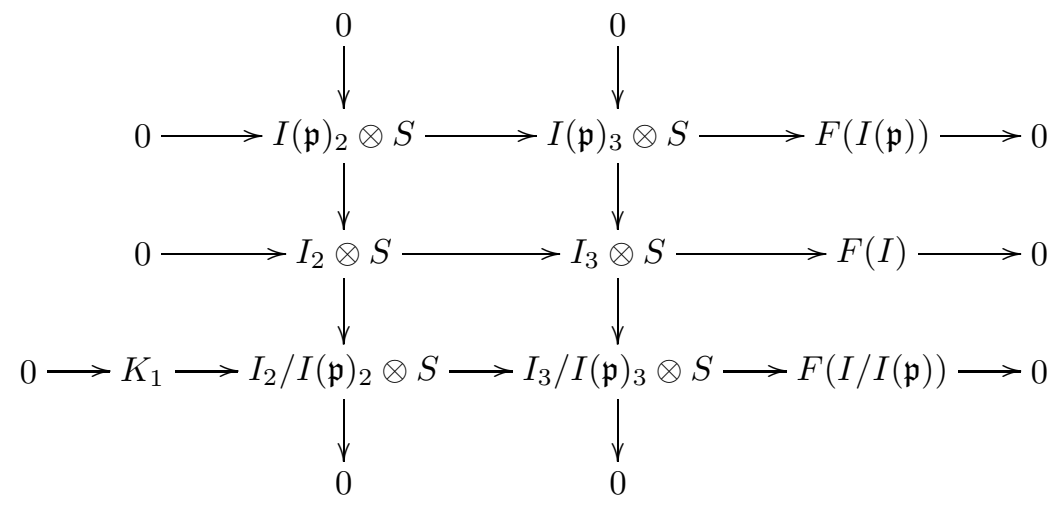

The Snake Lemma now yields the exact sequence

$$
0 \longrightarrow K_{1} \longrightarrow F(I(\mathfrak{p})) \longrightarrow F(I) \longrightarrow F(I / I(\mathfrak{p})) \longrightarrow 0 \text {. }
$$

All the $S$-modules in the two middle columns of diagram (5.4) are free. Thus, their duals are also free. Hence, dualizing the full diagram, we obtain the commuting diagram

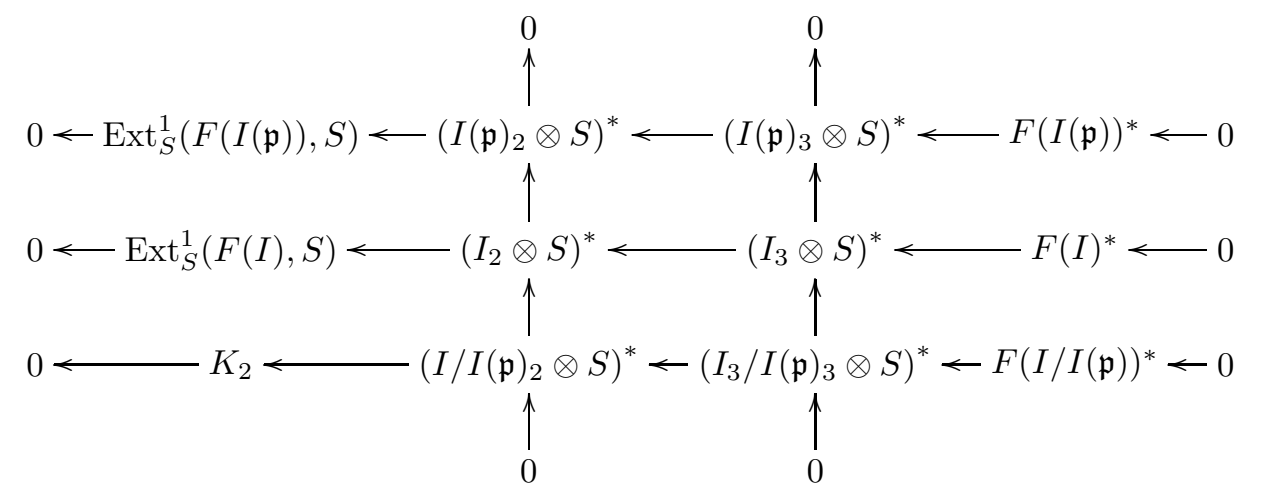


from which we derive, as before, an exact sequence

$$
\begin{aligned}
0 \rightarrow F(I / I(\mathfrak{p}))^{*} \rightarrow F(I)^{*} \longrightarrow F(I(\mathfrak{p}))^{*} \\
K_{2} \longrightarrow \operatorname{Ext}_{S}^{1}(F(I), S) \rightarrow \operatorname{Ext}_{S}^{1}(F(I(\mathfrak{p})), S) \rightarrow 0 .
\end{aligned}
$$

Let $\epsilon(\mathfrak{p}): \operatorname{Ext}_{S}^{1}(F(I), S) \rightarrow \operatorname{Ext}_{S}^{1}(F(I(\mathfrak{p})), S)$ be the surjective map on the right. By construction, the only associated prime of $B(\mathfrak{p})=\operatorname{Ext}_{S}^{1}(F(I(\mathfrak{p})), S)$ is $\mathfrak{p}$. Put $C=\operatorname{coker}\left(\bigoplus_{\mathfrak{p}} \epsilon(\mathfrak{p})\right)$. We obtain the exact sequence

$$
\operatorname{Ext}_{S}^{1}(F(I), S) \stackrel{\oplus_{\mathfrak{p}} \epsilon(\mathfrak{p})}{\longrightarrow} \bigoplus_{\mathfrak{p} \in \operatorname{Ass}\left(\mathcal{R}^{1}(\mathcal{A})\right)} \operatorname{Ext}_{S}^{1}(F(I(\mathfrak{p})), S) \longrightarrow C \longrightarrow 0
$$

Proposition 5.5. The $S$-module $C=\operatorname{coker}\left(\bigoplus_{\mathfrak{p}} \epsilon(\mathfrak{p})\right)$ is supported only at the maximal ideal $\mathfrak{m}$.

Proof. Localize the sequence (5.5) at a (minimal) prime $\mathfrak{q} \in \operatorname{Ass}\left(\mathcal{R}^{1}(\mathcal{A})\right)$. All the summands in the middle term disappear, except for $\mathfrak{B}(\mathfrak{q})=\operatorname{Ext}_{S}^{1}(F(I(\mathfrak{q})), S)$. This is because char $\mathbb{k}=0$, and so the components of $\mathcal{R}^{1}(\mathcal{A})$ are projectively disjoint; cf. 22. This gives an exact sequence

$$
\operatorname{Ext}_{S}^{1}(F(I), S)_{\mathfrak{q}} \stackrel{\epsilon(\mathfrak{q})_{\mathfrak{q}}}{\longrightarrow} \operatorname{Ext}_{S}^{1}(F(I(\mathfrak{q})), S)_{\mathfrak{q}} \longrightarrow C_{\mathfrak{q}} \longrightarrow 0
$$

Since localization is an exact functor, the surjection $\epsilon(\mathfrak{q})$ localizes to a surjection $\epsilon(\mathfrak{q})_{\mathfrak{q}}$. Thus, $C_{\mathfrak{q}}=0$.

If $\mathfrak{q}$ were an embedded prime for $\mathcal{R}^{1}(\mathcal{A})$, different from $\mathfrak{m}$, then the same argument as above shows that $C_{\mathfrak{q}}=0$. This finishes the proof.

5.3. A sharp inequality for $\theta_{k}$. We are now in position to prove one direction of Conjecture $\mathrm{A}$, Let $G$ be an arrangement group.

Theorem 5.6. For $k$ sufficiently large,

$$
\theta_{k}(G) \geq(k-1) \sum_{\mathfrak{p} \in \operatorname{Ass}\left(\mathcal{R}^{1}(\mathcal{A})\right)}\left(\begin{array}{c}
\operatorname{dim} L_{\mathfrak{p}}+k-1 \\
k
\end{array}\right) .
$$

Proof. Let $K=\operatorname{ker}\left(\bigoplus_{\mathfrak{p}} \epsilon(\mathfrak{p})\right)$. We have an exact sequence

$$
0 \rightarrow K \rightarrow \operatorname{Ext}_{S}^{1}(F(I), S) \stackrel{\oplus_{\mathfrak{p}} \epsilon(\mathfrak{p})}{\longrightarrow} \bigoplus_{\mathfrak{p} \in \operatorname{Ass}\left(\mathcal{R}^{1}(\mathcal{A})\right)} \operatorname{Ext}_{S}^{1}(F(I(\mathfrak{p})), S) \rightarrow C \rightarrow 0
$$

By Theorem 2.2 and Proposition 5.5, we see that for $k \gg 0$,

$$
\begin{aligned}
\theta_{k}(G) & =\mathrm{P}(\mathfrak{B}, k) \\
& =\sum_{\mathfrak{p} \in \operatorname{Ass}\left(\mathcal{R}^{1}(\mathcal{A})\right)} \mathrm{P}\left(\operatorname{Ext}_{S}^{1}(F(I(\mathfrak{p})), S), k\right)+\mathrm{P}(K, k) .
\end{aligned}
$$

By Corollary 5.4,

$$
\mathrm{P}\left(\operatorname{Ext}_{S}^{1}(F(I(\mathfrak{p})), S), k\right)=(k-1)\left(\begin{array}{c}
\operatorname{dim} L_{\mathfrak{p}}+k-1 \\
k
\end{array}\right) .
$$

Combining equations (5.7) and (5.8) yields the desired inequality. 
Theorem 5.6 gives the lower bound predicted by the Chen ranks conjecture. Proving the other direction of the inequality is equivalent to showing that the module $K$ is of finite length. While localization techniques should still be useful here, the problem seems more delicate. In the next section, we will indicate another possible way to attack this problem.

\section{Discussion And EXAMPles}

6.1. An alternate approach. Let $\mathcal{A}$ be an arrangement in $\mathbb{C}^{3}$, and let $\mathfrak{p} \in$ $\operatorname{Ass}\left(\mathcal{R}^{1}(\mathcal{A})\right)$ be one of the minimal prime ideals associated to the resonance variety $\mathcal{R}^{1}(\mathcal{A})$. Recall we defined in (4.2) and (5.2) two $S$-modules:

$$
M(\mathfrak{p})=\{x \in \mathfrak{B} \mid \operatorname{ann}(x)=\mathfrak{p}\} \quad \text { and } \quad \mathfrak{B}(\mathfrak{p})=\operatorname{Ext}_{S}^{1}(F(I(\mathfrak{p})), S) .
$$

Computations suggest that $M(\mathfrak{p})$ and $\mathfrak{B}(\mathfrak{p})$ define the same sheaf. Proving that $\widetilde{M}(\mathfrak{p})=\widetilde{\mathfrak{B}}(\mathfrak{p})$ would imply, via (4.4), the Chen ranks conjecture. If we put

$$
\mathfrak{B}^{\prime}=\bigoplus_{\mathfrak{p} \in \operatorname{Ass}\left(\mathcal{R}^{1}(\mathcal{A})\right)} \mathfrak{B}(\mathfrak{p})
$$

then $\widetilde{M}(\mathfrak{p})=\widetilde{\mathfrak{B}}(\mathfrak{p})$ would also imply that

$$
\mathfrak{B}^{\prime}=\sum_{v \in \mathbb{Z}} H^{0}(\widetilde{\mathfrak{B}}(v)) .
$$

6.2. Almost neighborly partitions. The decomposable arrangements studied in [7] have only local resonance. For these arrangements, $\mathfrak{B} \cong \mathfrak{B}^{\prime}$, and it follows easily that $H_{\mathfrak{m}}^{0}(\mathfrak{B})$ and $H_{\mathfrak{m}}^{1}(\mathfrak{B})$ vanish. We note that while $H_{\mathfrak{m}}^{0}(\mathfrak{B})$ and $H_{\mathfrak{m}}^{1}(\mathfrak{B})$ are not important for computing the asymptotic Chen ranks, the next examples indicate that they do capture interesting combinatorial features of an arrangement.

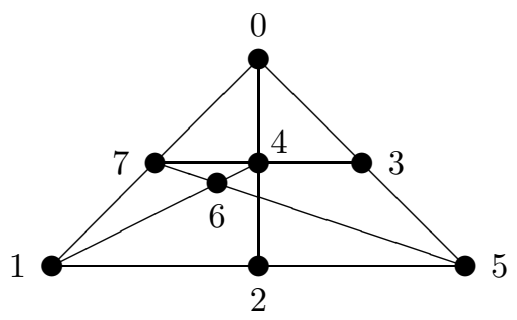

Figure 2. The deleted MacLane matroid

Example 6.1. The deleted MacLane arrangement corresponds to a matroid on 8 points, obtained by deleting a line from the $\mathrm{ML}_{8}$ matroid; see Figure 2. The resonance variety has 7 local components, corresponding to the triple points. A computation shows:

\begin{tabular}{c|cccc}
$k$ & $\operatorname{dim}_{\mathbb{k}} H_{\mathfrak{m}}^{0}(\mathfrak{B})_{k}$ & $\operatorname{dim}_{\mathbb{k}} \mathfrak{B}_{k}$ & $\operatorname{dim}_{\mathbb{k}} \mathfrak{B}_{k}^{\prime}$ & $\operatorname{dim}_{\mathbb{k}} H_{\mathfrak{m}}^{1}(\mathfrak{B})_{k}$ \\
\hline 2 & 0 & 7 & 7 & 0 \\
3 & 1 & 15 & 14 & 0 \\
4 & 0 & 21 & 21 & 0
\end{tabular}

Moreover, $\operatorname{Hilb}(\mathfrak{B})=\frac{7 t^{2}}{(1-t)^{2}}+t^{3}=7 t^{2}+15 t^{3}+21 t^{4}+28 t^{5}+\cdots$, and so $\theta_{k}=7(k-1)$, for $k \geq 4$. 
The interesting feature in the above example is the jump of 1 in the dimension of $\mathfrak{B}_{3}$ (when compared to that of $\mathfrak{B}_{3}^{\prime}$ ), coming from the non-vanishing of $H_{\mathfrak{m}}^{0}(\mathfrak{B})_{3}$. This jump can be explained by the partition (06|13|27|45), which is "almost" neighborly, though not neighborly.

Definition 6.2. A partition $\Pi$ of $\mathcal{A}$ is almost neighborly if, for any rank two flat $Y \in L_{2}(\mathcal{A})$ with $\mu(Y)>1$ and any block $\pi$ of $\Pi$,

$$
\mu(Y) \leq|Y \cap \pi| \Longrightarrow Y \subseteq \pi
$$

While such partitions cannot contribute to resonance (at least not in characteristic 0), it seems that they manifest their presence in the free resolution of $A$ over $E$. Computations indicate that non-vanishing $H_{\mathfrak{m}}^{0}(\mathfrak{B})$ is often linked to the existence of almost neighborly partitions. Do there exist examples where $H_{\mathfrak{m}}^{0}(\mathfrak{B})$ is nonzero, but which have no almost neighborly partitions?

6.3. Regularity and asymptotic range. The fact that the module $\mathfrak{B}$ may not be saturated means that the problem of determining for which $k$ the asymptotic values for $\theta_{k}$ given by Conjecture $\mathrm{A}$ are valid is rather subtle. In particular, the regularity of $\mathfrak{B}$ is related to the homology of the linear strand of $A$ over $E$ (see [13], Corollary 2.4), and will be influenced by the non-vanishing of $H_{\mathfrak{m}}^{0}(\mathfrak{B})$.

Regularity also influences the values for which the Hilbert polynomial and Hilbert function agree (see [32, Corollary B.4.1). This is illustrated in the next example.

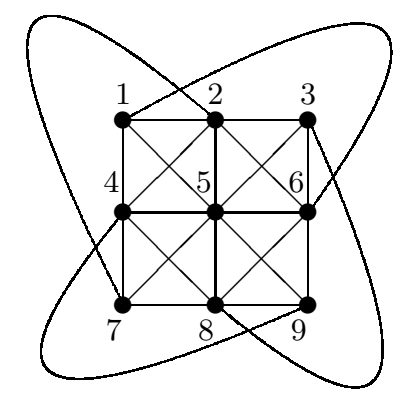

Figure 3. The Ceva(3) matroid

Example 6.3. The Ceva(3) arrangement (also known as the monomial arrangement $\mathcal{A}(3,3,3))$, is a realization of the affine plane over $\mathbb{Z}_{3}$, with defining polynomial $Q=\left(x^{3}-y^{3}\right)\left(x^{3}-z^{3}\right)\left(y^{3}-z^{3}\right)$. Its matroid is depicted in Figure 3 . The resonance variety has 12 local components, corresponding to the triple points, and 4 essential, 1-dimensional components, corresponding to the neighborly partitions (123|456|789), (147|258|369), (159|267|348), (168|249|357). We compute:

\begin{tabular}{c|cccc}
$k$ & $\operatorname{dim}_{\mathbb{k}} H_{\mathfrak{m}}^{0}(\mathfrak{B})_{k}$ & $\operatorname{dim}_{\mathbb{k}} \mathfrak{B}_{k}$ & $\operatorname{dim}_{\mathbb{k}} \mathfrak{B}_{k}^{\prime}$ & $\operatorname{dim}_{\mathbb{k}} H_{\mathfrak{m}}^{1}(\mathfrak{B})_{k}$ \\
\hline 2 & 0 & 12 & 16 & 4 \\
3 & 8 & 40 & 32 & 0 \\
4 & 8 & 56 & 48 & 0 \\
5 & 0 & 64 & 64 & 0
\end{tabular}

Also, $\operatorname{Hilb}(\mathfrak{B})=\frac{16 t^{2}}{(1-t)^{2}}+4 t^{2}\left(2 t^{2}+2 t-1\right)=12 t^{2}+40 t^{3}+56 t^{4}+64 t^{5}+80 t^{6}+\cdots$, and so $\theta_{k}=16(k-1)$, for $k \geq 5$. 
As noted in 1.4, the original form of Conjecture $\mathrm{A}$ assumed that taking $k \geq 4$ would suffice to ensure equality. The non-vanishing of $H_{\mathfrak{m}}^{0}(\mathfrak{B})_{4}$ in Example 6.3 shows that a larger $k$ is needed, in general.

\section{ACKNOWLEDGEMENTS}

Computations using Macaulay 2 [20] were essential to our efforts. We are grateful to MSRI, where part of this work was performed. Thanks to the referee for a very careful reading of the manuscript, and for useful suggestions.

\section{REFERENCES}

[1] K. Aomoto, Un théorème du type de Matsushima-Murakami concernant l'intégrale des fonctions multiformes, J. Math. Pures Appl. 52 (1973), 1-11. MR0396563 (53:426)

[2] A. Aramova, L. Avramov, J. Herzog, Resolutions of monomial ideals and cohomology over exterior algebras, Trans. Amer. Math. Soc. 352 (1999), 579-594. MR 1603874 (2000c:13021)

[3] L. Avramov, Infinite free resolutions, Six lectures on commutative algebra (Bellaterra, 1996), 1-118, Progr. Math., 166, Birkhäuser, Basel, 1998. MR1648664 (99m:13022)

[4] K. T. Chen, Integration in free groups, Ann. of Math. 54 (1951), 147-162. MR0042414 $(13: 105 \mathrm{c})$

[5] D. Cohen, P. Orlik, Arrangements and local systems, Math. Res. Lett. 7 (2000), 299-316. MR1764324 (2001i:57040)

[6] D. Cohen, A. Suciu, The Chen groups of the pure braid group, The Cech centennial (Boston, MA, 1993), 45-64, Contemp. Math., 181, Amer. Math. Soc., Providence, RI, 1995. MR1320987 (96c:20055)

[7] , Alexander invariants of complex hyperplane arrangements, Trans. Amer. Math. Soc. 351 (1999), 4043-4067. MR1475679 (99m:52019)

[8] _ Characteristic varieties of arrangements, Math. Proc. Cambridge Phil. Soc. 127 (1999), 33-53. MR1692519 (2000m:32036)

[9] W. Decker, D. Eisenbud, Sheaf algorithms using the exterior algebra, in: Computations in Algebraic Geometry using Macaulay 2, Springer-Verlag, Berlin, Heidelberg, New York, 2002. MR.1949553

[10] G. Denham, S. Yuzvinsky, Annihilators of Orlik-Solomon relations, Adv. in Appl. Math. 28 (2002), 231-249. MR1888846 (2003b:05046)

[11] D. Eisenbud, Commutative algebra with a view towards algebraic geometry, Graduate Texts in Math., vol. 150, Springer-Verlag, Berlin, Heidelberg, New York, $1995 . \quad$ MR 1322960 (97a:13001)

[12] The geometry of syzygies, Springer-Verlag, Berlin, Heidelberg, New York, 2004. MR2103875

[13] D. Eisenbud, G. Fløystad, F.-O. Schreyer, Sheaf cohomology and free resolutions over exterior algebras, Trans. Amer. Math. Soc. 355 (2003), 4397-4426. MR.1990756 (2004f:14031)

[14] D. Eisenbud, S. Popescu, S. Yuzvinsky, Hyperplane arrangement cohomology and monomials in the exterior algebra, Trans. Amer. Math. Soc. 355 (2003), 4365-4383. MR1986506 (2004g:52036)

[15] H. Esnault, V. Schechtman, E. Viehweg, Cohomology of local systems on the complement of hyperplanes, Invent. Math. 109 (1992), 557-561. MR:1176205 (93g:32051)

[16] M. Falk, Arrangements and cohomology, Ann. Combin. 1 (1997), 135-157. MR1629681 (99g:52017)

[17] The line geometry of resonance varieties, preprint arXiv:math.C0/0405210.

[18] M. Falk, R. Randell, On the homotopy theory of arrangements, II, in: Arrangements-Tokyo 1998, Adv. Stud. Pure Math., vol. 27, Math. Soc. Japan, Kinokuniya, Tokyo, 2000, pp. 93125. MR:1796895 (2002b:32044)

[19] R. Fröberg, C. Löfwall, Koszul homology and Lie algebras with application to generic forms and points, Homology Homotopy Appl. 4 (2002), 227-258. MR1918511(2003g:13018)

[20] D. Grayson, M. Stillman, Macaulay 2: a software system for research in algebraic geometry; available at http://www.math.uiuc.edu/Macaulay2.

[21] H. Hamm, Lê D. T., Un théorème de Zariski du type de Lefschetz, Ann. Sci. École Norm. Sup. 6 (1973), 317-356. MR0401755 (53:5582) 
[22] A. Libgober, S. Yuzvinsky, Cohomology of the Orlik-Solomon algebras and local systems, Compositio Math. 121 (2000), 337-361. MR.1761630(2001j:52032)

[23] W.S. Massey, Completion of link modules, Duke Math. J. 47 (1980), 399-420. MR0575904 (81g:57004)

[24] D. Matei, A. Suciu, Cohomology rings and nilpotent quotients of real and complex arrangements, in: Arrangements-Tokyo 1998, Adv. Stud. Pure Math., vol. 27, Math. Soc. Japan, Tokyo, 2000, pp. 185-215. MR1796900 (2002b:32045)

[25] _ Hall invariants, homology of subgroups, and characteristic varieties, Int. Math. Res. Not. 2002, no. 9, 465-503. MR1884468 (2003d:20055)

[26] P. Orlik, L. Solomon, Combinatorics and topology of complements of hyperplanes, Invent. Math. 56 (1980), 167-189. MR0558866 (81e:32015)

[27] P. Orlik, H. Terao, Arrangements of hyperplanes, Grundlehren Math. Wiss., vol. 300, Springer-Verlag, New York, Berlin, Heidelberg, 1992. MR217488 (94e:52014)

[28] S. Papadima, A. Suciu, Chen Lie algebras, Int. Math. Res. Not. 2004:21 (2004), 1057-1086. MR2037049(2004m:17043)

[29] - When does the associated graded Lie algebra of an arrangement group decompose?, preprint arXiv:math.CO/0309324.

[30] H. Schenck, A. Suciu, Lower central series and free resolutions of hyperplane arrangements, Trans. Amer. Math. Soc. 354 (2002), 3409-3433. MR1911506 (2003k:52022)

[31] A. Suciu, Fundamental groups of line arrangements: Enumerative aspects, in: Advances in algebraic geometry motivated by physics, Contemporary Math., vol. 276, Amer. Math. Soc, Providence, RI, 2001, pp. 43-79. MR.1837109 (2002k:14029)

[32] W. Vasconcelos, Computational methods in commutative algebra and algebraic geometry, Springer-Verlag, Berlin, Heidelberg, New York, 1998. MR.1484973 (99c:13048)

[33] S. Yuzvinsky, Cohomology of Brieskorn-Orlik-Solomon algebras, Comm. Algebra 23 (1995), 5339-5354. MR1363606 (97a:52023)

[34] Orlik-Solomon algebras in algebra and topology, Russ. Math. Surveys 56 (2001), 293-364. MR1859708 (2002i:14047)

Department of Mathematics, Texas A\&M University, College Station, Texas 77843

E-mail address: schenck@math.tamu.edu

URL: http://www. math.tamu.edu/ ${ }^{\text {schenck }}$

Department of Mathematics, Northeastern University, Boston, Massachusetts 02115

E-mail address: a.suciu@neu.edu

$U R L:$ http://www.math.neu.edu/〜 suciu 